\title{
Care in Chronic Obstructive Lung Disease (CAROL): a randomised trial in general practice
}

\author{
Stefan Markun', Thomas Rosemann ${ }^{1}$ Kaba Dalla-Lana² and \\ Claudia Steurer-Stey ${ }^{2}$
}

Affiliations: ${ }^{1}$ Institute of Primary Care, University and University Hospital of Zurich, Zurich, Switzerland. ${ }^{2}$ Epidemiology, Biostatistics and Prevention Institute, University of Zurich, Zurich, Switzerland.

Correspondence: Stefan Markun, Institute of Primary Care, Pestalozzistrasse 24, 8091 Zurich, Switzerland. E-mail: stefan.markundusz.ch

@ERSpublications

Disease management using a care bundle increases guideline adherence in general practice care for COPD http://ow.ly/E6b930jlO7y

Cite this article as: Markun S, Rosemann T, Dalla-Lana K, et al. Care in Chronic Obstructive Lung Disease (CAROL): a randomised trial in general practice. Eur Respir J 2018; 51: 1701873 [https://doi.org/ 10.1183/13993003.01873-2017].

ABSTRACT Disease management of chronic obstructive pulmonary disease (COPD) is complex and shortcomings in general practice care for COPD are common. A care bundle is a disease management aid used as a reminder and for steering specific elements of care. Our objectives were to test whether a COPD care bundle delivered to general practitioners (GPs) and practice assistants increases the implementation of key elements of COPD care.

The study was a cluster-randomised clinical trial, with 1:1 randomisation of GPs and a 1-year follow-up. The intervention introduced a COPD care bundle and aimed at enhancing collaboration between GPs and practice assistants. The control group continued usual care. The primary outcome measure was the composite score from nine key elements of COPD care measured at the patient level.

We enrolled 35 GPs and 216 patients with a median age of 69 years, 59\% female, $69 \%$ Global Initiative for Chronic Obstructive Lung Disease group A or B. After 1 year, the between-group difference in change of the primary outcome measure was $+2.2(95 \% \mathrm{CI}+1.5-+2.9)$ in favour of the intervention group. The intervention was associated with significantly higher implementation rates in seven out of nine key elements of care.

Disease management using a COPD care bundle increased the implementation of key elements of COPD care in general practice. 


\section{Introduction}

Chronic obstructive pulmonary disease (COPD) is of high and increasing prevalence, and is an important contributor to worldwide years of life lost $[1,2]$. COPD is, however, a preventable disease, and modifiable risk factors and many effective interventions that reduce symptoms and improve prognosis have been identified. Guidelines amalgamate the existing evidence into practically applicable treatment recommendations $[3,4]$. Nonetheless, we continue to observe shortcomings in COPD care delivered in general practice [5-10]. This is of special concern because the majority of COPD patients are treated in general practice and are in early disease stages when preventive interventions have the most potential to improve outcomes [11-14].

Evidence-based care for COPD is complex because it is stage- and symptom-dependent and comprises multimodal interventions: disease assessment requires spirometry and collection of several variables (e.g. symptom severity and exacerbation history) to determine stage and treatment. Therapeutic measures with a robust evidence base (subsequently referred to as "key elements of COPD care") comprise smoking cessation [15], influenza vaccination [16], appropriate pharmacological therapy (also ensuring correct inhalation technique) [3, 17-21], pulmonary rehabilitation [22], sustaining physical activity [23], self-management education [24-26] and proactive, integrated disease management [27].

While some key elements of COPD care are straightforward to deliver, e.g. influenza vaccination, others require time, knowledge, skills, and interprofessional collaboration and coordination. The plethora of key elements and the individual implications of each one of them add up to a bundle of interventions that is complex to coordinate and deliver. This is critical, especially in general practice, where doctors struggle with putting into practice the broad and continuously expanding field of general medicine, and complex interventions are at risk of being left behind.

Organisational changes and structured disease management aids can facilitate implementation of complex care pathways. For COPD, such approaches have already been successfully tested in hospital medicine. So-called "COPD care bundles" have been used as reminder lists summarising key elements of care to be implemented at the individual patient level before hospital discharge. In hospital-based COPD care, care bundles succeeded in not only raising implementation rates of key elements of care but also in reducing readmission rates [28]. In primary care, COPD disease management trials are scarce. One trial aimed to increase implementation of best-practice guidelines through home visits from specifically trained nurses who developed individualised care plans with COPD patients and which resulted in improved quality of care [29]. A trial implementing a COPD management guideline, monthly nurse and 3-monthly GP visits, a patient-specific care plan and enhancing collaboration between healthcare providers resulted in reduced hospital admissions, less hospital days and increased implementation of some key elements of care [30]. However, the intensive and multimodal interventions of previous trials render attribution of the identified effects to individual intervention components difficult.

The aim of this trial was to test whether an intervention focusing on general practice teams including implementation of a COPD care bundle along with specific coaching to support organisational and behavioural changes would result in an increased implementation rate of key elements of COPD care.

\section{Methods}

Study design, setting, registration and ethics statement

We conducted a parallel group cluster-randomised trial with general practices working in the Swiss canton of Zurich. The local ethics committee approved the study (Ethics Committee of the Canton of Zurich; KEK-ZH 2013-0189), informed consent was retrieved from all participating subjects, and the study was conducted according to the tenets of the Declaration of Helsinki and good clinical practice guidelines. The trial was registered at ClinicalTrials.gov (identifier NCT01921556) and the trial's study protocol has been published [31].

\section{Participants}

Recruitment of general practitioners (GPs) started in 2013 by mass mailings and visits at GP network meetings. We enrolled 35 GPs after a 9-month GP recruitment period. We trained GPs and their practice assistants in standardised spirometry to enhance accuracy of diagnostic testing for COPD. Patient recruitment started in December 2013. A detailed report of the trial's recruitment period has been published [12].

Eligibility criteria for GPs were: 1) primary care physician in the canton of Zurich and 2) board certification in general medicine or internal medicine. GPs approached consecutive patients aged at least 45 years, with at least 10 pack-years smoking history, and proposed to perform spirometry. If airflow obstruction (forced expiratory volume in 1 s/forced vital capacity <0.7) was confirmed, GPs gained 
informed consent if available and performed formal study inclusion. Exclusion criteria for patients were: emergency consultations, insufficient German language skills to complete study questionnaires, asthma or hay fever, or estimated life expectancy $<6$ months.

\section{Data collection}

Data was collected using self-administered questionnaires (see supplementary material) at patient recruitment ( $\left.\mathrm{T}_{0}\right)$ and 12 months after the intervention ( $\left.\mathrm{T}_{1}\right)$. GPs completed a questionnaire about their own demographic characteristics and working environment. We pilot-tested the patient questionnaire with six COPD patients from the targeted group and made according adjustments to improve comprehensibility. The questionnaire asked for sociodemographic data, smoking status, 12-month retrospective view on delivered key elements of care (see later) and symptoms, including the COPD Assessment Test (CAT). GPs filled in a questionnaire that asked for anthropometric patient data, including current spirometry results, 12-month retrospective COPD exacerbations and COPD-driven health service utilisation as well as prescribed pulmonary drugs. Table 1 shows the measured key elements of care including the levels of measurements and the applicable patient subgroups.

\section{Intervention}

We delivered the intervention after the patient recruitment period in a half-day workshop with GPs and their practice assistants. The intervention aimed at implementing the COPD care bundle and inducing organisational and behavioural changes in the general practice teams. First, we refreshed knowledge about Swiss COPD guidelines [4] and distributed a pocket guide. Then, GPs and practice assistants were to discuss and tailor their individual pathways of COPD care. Case vignettes and role plays were used to actively involve GPs and practice assistants with tasks and responsibilities. We proposed to use the COPD care bundle as a checklist to remind and tick-off the individual key elements of COPD care in individual patients. We expected the care bundle's design as a checklist to increase internal motivation for behaviour change $[32,33]$. We delivered no intervention to the "usual care" control group.

After 6 months, we delivered a 3-h refresher workshop to the practice teams again using case vignettes and role plays after conducting a survey among practice teams to inform us about their specific needs for support.

\section{Outcomes}

Primary outcome

Between-group difference in the change of implemented key elements of COPD care after 1 year (see table 1).

\begin{tabular}{|c|c|c|c|}
\hline Key elements of COPD care & $\begin{array}{l}\text { Applicable patient } \\
\text { subgroup }\end{array}$ & $\begin{array}{l}\text { Component of } \\
\text { outcome }\end{array}$ & $\begin{array}{l}\text { Measurement } \\
\text { level }\end{array}$ \\
\hline 1 Smoking cessation advice & Smokers only & Primary & Patient \\
\hline 2 Smoking cessation intervention & Smokers only & Primary & Patient \\
\hline 3 Influenza vaccination & All patients & Primary & Patient \\
\hline $\begin{array}{l}4 \text { Ensuring correct inhalation } \\
\text { technique }\end{array}$ & All patients & Primary & Patient \\
\hline $\begin{array}{l}5 \text { Appropriate pharmacological } \\
\text { treatment }\end{array}$ & All patients & Primary & GP \\
\hline 6 Assessment of physical activity & All patients & Primary & Patient \\
\hline 7 Advice for physical activity & All patients & Primary & Patient \\
\hline 8 Patient education class referral & All patients & Primary & Patient \\
\hline $\begin{array}{l}9 \text { Assessment of exacerbation } \\
\text { frequency }\end{array}$ & All patients & Primary & GP \\
\hline $\begin{array}{l}10 \text { Integration of other healthcare } \\
\text { providers }\end{array}$ & GOLD $C$ and $D$ patients & Secondary & Patient \\
\hline $\begin{array}{l}11 \text { Referral to pulmonary } \\
\text { rehabilitation }\end{array}$ & GOLD $C$ and $D$ patients & Secondary & Patient \\
\hline 12 Exacerbation action plan & GOLD $C$ and $D$ patients & Secondary & Patient \\
\hline
\end{tabular}

GP: general practitioner; GOLD: Global Initiative for Chronic Obstructive Lung Disease. * : explanation, demonstration and assessment of patient's inhaler technique. 
Secondary outcomes

1) Between-group difference in proportions of Global Initiative for Chronic Obstructive Lung Disease (GOLD) group C or D patients who received referral to pulmonary rehabilitation, a written action plan for exacerbation management or coordinated care.

2) Between-group difference in symptom severity measured with the CAT instrument.

\section{Sample size}

Based on available data from Switzerland $[5,8]$, we assumed a mean \pm SD of $4 \pm 2.3$ implemented key elements of COPD care. We assumed a 1.5-point increase to be a relevant improvement and used this difference to calculate the sample size. Given a power of $90 \%$ and a significance level $\alpha$ of $5 \%$, as well as an intracluster correlation coefficient of 0.04 , we targeted at recruiting $30 \mathrm{GPs}$ each recruiting eight patients, resulting in 240 patients. To allow for dropout we set a recruitment target of 35 GPs.

\section{Randomisation}

The level of randomisation was the individual GP and the allocation ratio was 1:1. We performed randomisation of GPs 6 months after initiation of patient recruitment to minimise the effect of the openly labelled treatment group allocation on recruitment performance. To balance groups for the considerable variation in recruiting performance, we ranked GPs according to their number of recruited patients and assigned random group allocation with a block size of 2 . A researcher not involved in this study produced

\begin{tabular}{|c|c|c|c|}
\hline & Intervention group & Control group & p-value \\
\hline Patients & 101 & 115 & \\
\hline Age years & $68(63-75)$ & $67(60-73)$ & 0.260 \\
\hline Male & $60(59.4)$ & $68(59.1)$ & 0.967 \\
\hline BMI $\mathrm{kg} \cdot \mathrm{m}^{-2}$ & $25.9 \pm 5.99$ & $25.6 \pm 4.63$ & 0.753 \\
\hline \multicolumn{4}{|l|}{ GOLD group $\pi$} \\
\hline$A$ & $68(67.3)$ & 59 (51.3) & 0.101 \\
\hline $\mathrm{B}$ & $9(8.9)$ & 13 (11.3) & \\
\hline $\mathrm{C}$ & $16(15.8)$ & $25(21.7)$ & \\
\hline $\mathrm{D}$ & $8(7.9)$ & $18(15.7)$ & \\
\hline FEV $1 \%$ pred & $70(55-86)$ & $65(51-76)$ & 0.035 \\
\hline$\geqslant 1$ exacerbations in past 12 months & $27(26.7)$ & $46(40.0)$ & 0.089 \\
\hline New COPD diagnosis at recruitment & $37(36.6)$ & $34(29.6)$ & 0.270 \\
\hline Composite score of implemented key elements of care ${ }^{+}$ & $4.1 \pm 2.0$ & $4.6 \pm 1.7$ & 0.035 \\
\hline CAT summary score & $9(6-15)$ & $12(8-16)$ & 0.033 \\
\hline \multicolumn{4}{|l|}{ mMRC category } \\
\hline 0 & $27(27.3)$ & $25(22.7)$ & 0.904 \\
\hline 1 & $42(42.4)$ & $48(43.6)$ & \\
\hline 2 & $23(23.2)$ & $28(25.5)$ & \\
\hline 3 & $7(7.1)$ & $9(8.2)$ & \\
\hline 4 & $0(0)$ & $4(3.6)$ & \\
\hline Active smokers & $56(55.4)$ & 64 (55.7) & 0.976 \\
\hline Pack-years & $44(30-59)$ & $45(35-60)$ & 0.277 \\
\hline Diabetes & $14(14.0)$ & $14(12.6)$ & 0.767 \\
\hline Hypertension & 50 (51.0) & 63 (55.3) & 0.537 \\
\hline Coronary heart disease & $16(16.3)$ & $22(19.6)$ & 0.533 \\
\hline Congestive heart failure & $12(12.0)$ & $9(8.0)$ & 0.335 \\
\hline Depression & $19(19.8)$ & $23(20.5)$ & 0.894 \\
\hline Follow-up days & 410 (398-428) & $440.5(410-481)$ & $<0.001$ \\
\hline
\end{tabular}

Data are presented as $\mathrm{n}$, median (interquartile range), $\mathrm{n}(\%)$ or mean $\pm \mathrm{SD}$, unless otherwise stated. BMI: body mass index; GOLD: Global Initiative for Chronic Obstructive Lung Disease; FEV1: forced expiratory volume in $1 \mathrm{~s}$; COPD: chronic obstructive pulmonary disease; CAT: COPD Assessment Test; mMRC: modified Medical Research Council. ${ }^{\#}$ : $\mathrm{n}=216$; ${ }^{\text {I: }}$ groups are classified according to the 2017 GOLD report $[3] ;{ }^{+}$: composite score being the sum of all implemented key elements ranging from 0 to 9 in smokers and 0 to 7 in nonsmokers (this score at patient recruitment comprises both patients with and without previously diagnosed COPD, and is therefore not to be understood as a measure for usual care in general practice COPD care). 
the random sequence using Stata (StataCorp, College Station, TX, USA). This randomisation method was applied to minimise risk of imbalanced allocation counts due to differences in recruiting performance. Furthermore, it balanced GPs for the possible confounding effect originating from the motivation to contribute to the trial, which we assumed to be associated with recruiting performance. We communicated the group allocations to GPs with the instruction not to pass this information to their patients. Patients were, however, aware that their GP would either continue usual care or start an experimental, potentially more comprehensive COPD care programme.

\section{Statistical methods}

We report counts and proportions for categorical data as well as mean and standard deviation or median and interquartile range (IQR) as appropriate. For bivariate group comparisons, we used a Welch test or a Wilcoxon rank sum test for continuous data and a Chi-squared test for categorical data and report p-values. The primary outcome was calculated with a linear regression model. The primary outcome measure at $\mathrm{T}_{1}$ was the dependent variable; independent variables were the group allocation as well as the following adjustment variables to minimise confounding: count of implemented processes at $\mathrm{T} 0$, patient age, sex, education (years), COPD stage and study follow-up time (days). We report the estimated between-group differences and the according 95\% confidence intervals. In a separate analysis we assessed for a cluster effect by adding the cluster variable (individual GPs) to the abovementioned regression model under a random effects assumption. We made no adjustments for a potential contamination effect originating from GPs in different study arms but located within the same group practice (therefore accepting a risk of underestimating the between-group difference in the trial results). To assess for selective dropout we analysed for between-group differences in counts and reasons for dropout. To assess the robustness of our results we carried out sensitivity analyses simulating missing data under several assumptions (multiple imputation method, last observation carried forward and imputing the average score of the control group). Statistical analysis was performed using R version 3.2.0 (www.R-project.org).

\section{Results}

\section{Study population}

Of the 35 GPs entering patient recruitment, 33 started recruiting and two withdrew before randomisation; therefore, 33 GPs were randomised (16 in the intervention group and 17 in the control group). 18 GPs (contributing 111 patients) from the intervention and control groups were colocated in group practices. During the 1-year recruitment period, GPs recruited 216 patients ( $90 \%$ of recruitment goal) starting in December 2013. Recruitment stopped when the number of newly recruited patients per month was $<5$. The study intervention was delivered in January 2015 and follow-up measures were conducted in January 2016. The patients had a median age of 69 years, 59\% were female and $69 \%$ GOLD group A or B. Per chance, the intervention group had less severe obstruction (median FEV1 \% pred $70 \%$ versus 65\%; $\mathrm{p}=0.035$ ) and a lower CAT summary score (median 9 versus 12; $\mathrm{p}=0.033$ ). Tables 2 and 3 give detailed patient and GP characteristics, including study group comparisons.

At $\mathrm{T} 1,161$ patients completed the follow-up (dropout rate 25\%) and the study ended as set out in the protocol. Figure 1 depicts patient and cluster recruitment and retention over the trial periods. When testing

\begin{tabular}{|c|c|c|c|}
\hline Variable & $\begin{array}{l}\text { Intervention } \\
\text { group }\end{array}$ & $\begin{array}{l}\text { Control } \\
\text { group }\end{array}$ & p-value \\
\hline GPs & $16(100)$ & $17(100)$ & \\
\hline Age years & $50(44-59)$ & $47(42-56)$ & 0.407 \\
\hline Male & $13(81.2)$ & $11(70.6)$ & 0.438 \\
\hline Single practice & $2(12.5)$ & $2(11.8)$ & 1.000 \\
\hline Group practice & $14(87.5)$ & $15(88.2)$ & 1.000 \\
\hline Electronic medical record & $13(81.2)$ & 13 (76.5) & 1.000 \\
\hline Paper-based medical record & $3(18.8)$ & $4(23.5)$ & 1.000 \\
\hline $\begin{array}{l}\text { Practice assistants workforce-equivalents in full-time } \\
\text { jobs } n\end{array}$ & $2.3(1.9-3.4)$ & $2.7(1.8-4.0)$ & 0.773 \\
\hline Estimated patients seen per day $n$ & $25(20-30)$ & $25(20-30)$ & 0.581 \\
\hline Patients approached $\mathrm{n}$ & $11(4-19)$ & $10(8-17)$ & 0.914 \\
\hline Patients recruited $\mathbf{n}$ & $6(2-10)$ & $6(5-10)$ & 0.638 \\
\hline
\end{tabular}

Data are presented as median (interquartile range) or $n(\%)$, unless otherwise stated. ${ }^{\#}: n=33$. 


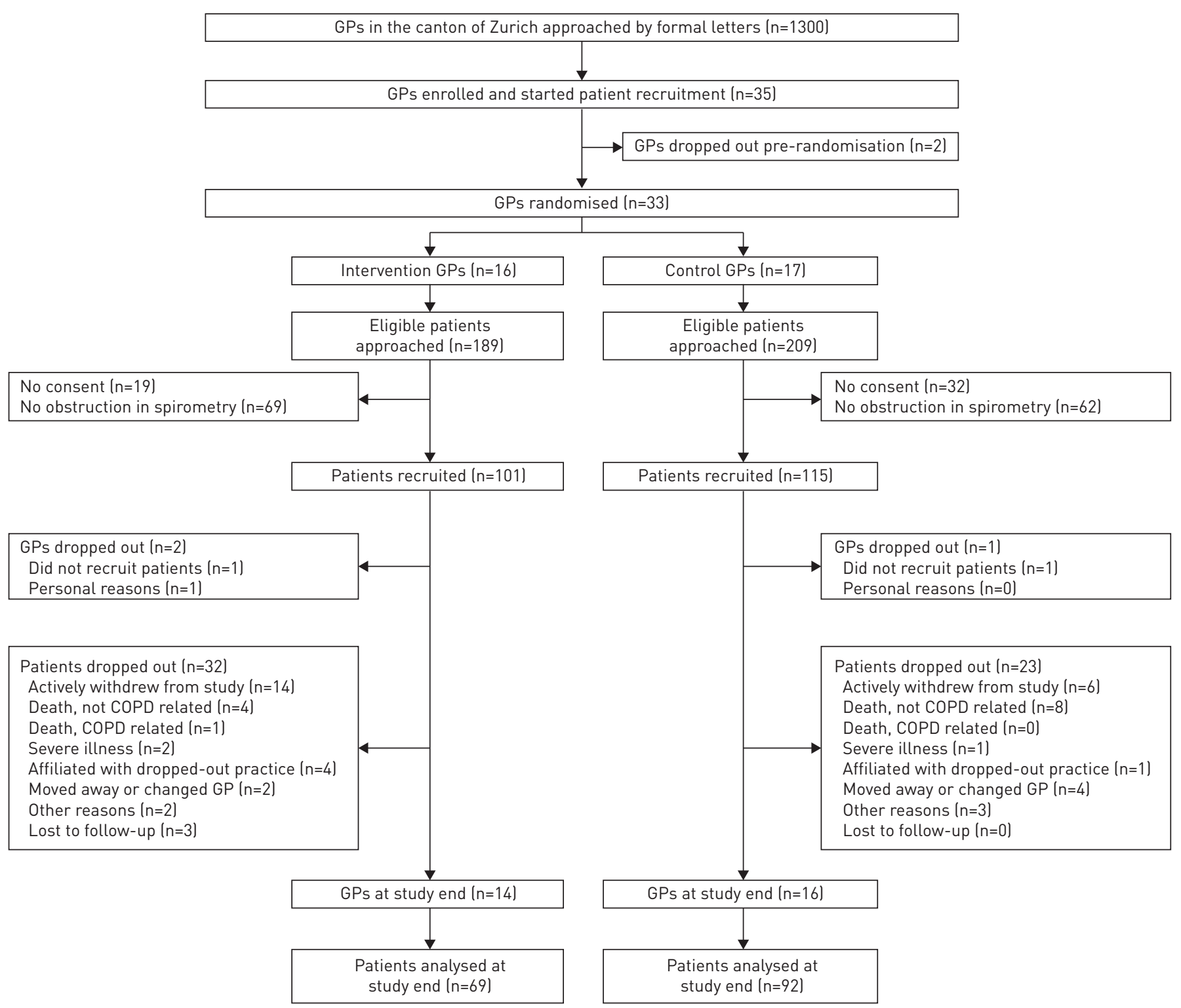

FIGURE 1 Flowchart of the study. GP: general practitioner; COPD: chronic obstructive pulmonary disease.

TABLE 4 Coefficients of the primary outcome's linear regression model

\begin{tabular}{lccc} 
& Estimate & Standard error & $\mathbf{9 5 \%} \mathbf{~ C l}$ \\
\hline Intervention group (reference: control group) & 2.2 & 0.64 & $1.5-2.9$ \\
Primary outcome at To & 0.4 & 0.38 & $0.2-0.6$ \\
Age & 0.0 & 0.11 & $-0.1-0$ \\
Sex (reference: female) & 0.2 & 0.02 & $-0.5-0.9$ \\
10-12 years education (reference: <12) & -0.5 & 0.36 & $-1.2-0.3$ \\
श13 years education (reference: <12) & 0.2 & 0.38 & $-0.7-1.1$ \\
Exacerbations in 1 year & 0.0 & 0.46 & $-0.2-0.2$ \\
FEV1\% pred & 0.0 & 0.09 & $0-0$ \\
CAT summary score at T1 & 0.0 & 0.00 & $0-0.1$ \\
Follow-up time & 0.0 & 0.01 & $0-0$ \\
\hline
\end{tabular}

FEV1: forced expiratory volume in $1 \mathrm{~s} ;$ CAT: COPD Assessment Test; T0: patient recruitment; T1: 12 months after the intervention. 
dropout counts, a significant between-group difference appeared (intervention group $\mathrm{n}=32$ versus control group $n=23 ; p=0.049$ ). Active withdrawal of patients was the most common reason for discontinuation; however, there was no significant between-group difference in reasons for discontinuation $(\mathrm{p}=0.165)$.

\section{Primary outcome}

After 1 year, the mean composite score of implemented key elements changed from 4.1 to $5.1(+1.0)$ in the intervention group and from 4.6 to $3.5(-1.1)$ in the control group. A linear regression model adjusting for baseline characteristics (table 4) revealed a between-group difference of $+2.2(95 \% \mathrm{CI}+1.5-+2.9)$ implemented key elements in favour of the intervention group. Significantly increased implementation was found in seven out of nine individual key elements (figure 2). We detected no significant cluster effect originating from individual GPs.

\section{Secondary outcomes}

In GOLD group C and D patients ( $\mathrm{n}=67(31 \%)$ ), no significant between-group difference appeared in the outcomes: integration of other healthcare providers, referral to pulmonary rehabilitation or delivery of exacerbation action plans (figure 2).

After 1 year, the mean CAT summary score decreased from 10.7 to $9.5(-1.2)$ in the intervention group and increased from 12.8 to $13.9(+1.1)$ in the control group. Linear regression model adjusting for baseline disparities showed an estimated difference in change of $-1.1(95 \%$ CI $-3.3+1.1 ; p=0.32)$ in the intervention group.

\section{Additional analyses}

Regarding intervention effects on individual key elements of COPD care, we identified different patterns. Implementation of certain key elements primarily increased in the intervention group (i.e. smoking cessation intervention, inhalation technique and patient education), while in other key elements between-group differences were primarily due to attrition in the control group (i.e. smoking cessation advice, physical activity assessment and advice). Figure 3 illustrates net differences of implementation rates between $\mathrm{T}_{0}$ and $\mathrm{T} 1$ per studied group.

The intervention effect on the primary outcome was stable and remained relevant in all sensitivity analyses: multiple imputation method (imputed datasets $\mathrm{n}=5)$ between-group difference of +1.6 ( $95 \% \mathrm{CI}$ $+0.8-+2.4)$; last observation carried forward method: $+2.3(95 \% \mathrm{CI}+1.5-+3.1)$; imputation of control group average: $+2.0(95 \% \mathrm{CI}+1.3-+2.8)$.

To further explore the adoption of the intervention, we asked the GPs in the intervention group how they implemented the COPD care bundle in the T1 questionnaire. In 47 (69\%) patients, the GPs used the care bundle as the intended checklist to complete, but in nine (13\%) as a recall list only and in $12(18 \%)$ the

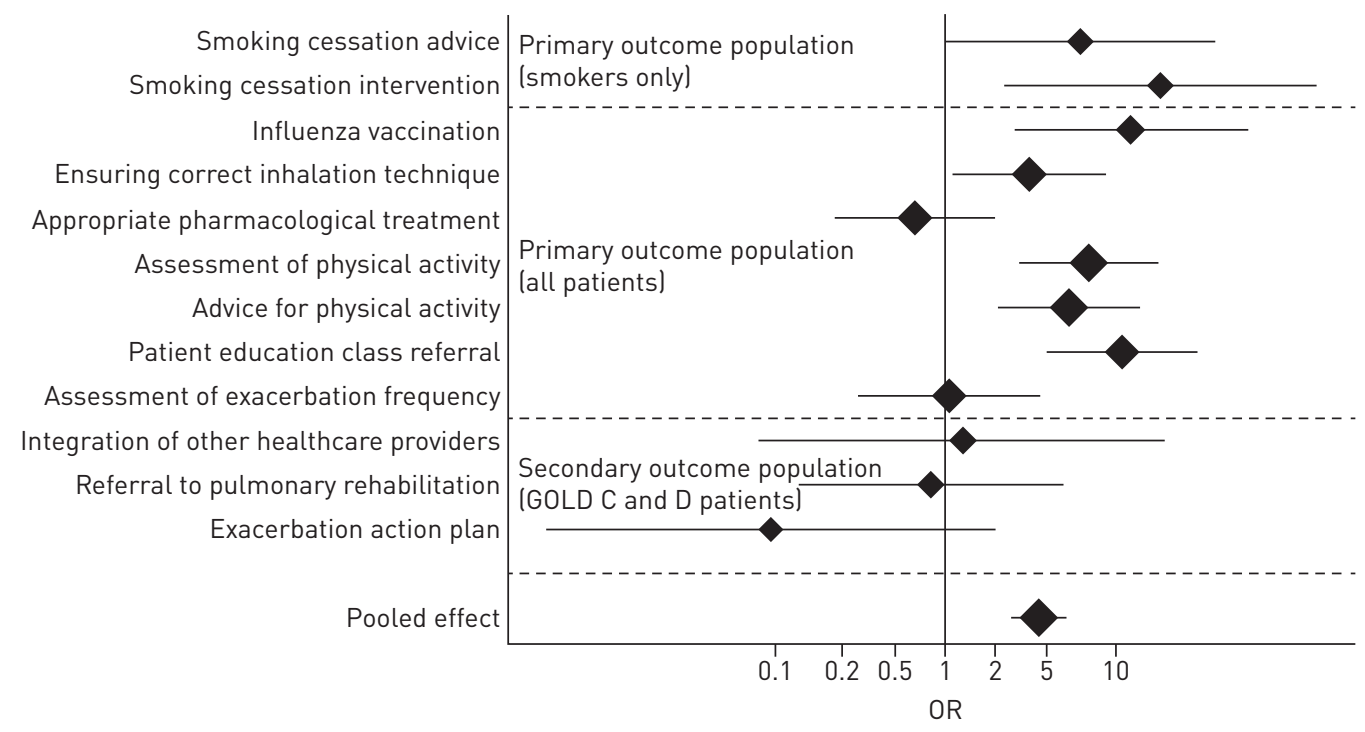

FIGURE 2 Intervention effects on implementation rates of individual key elements of chronic obstructive pulmonary disease care. GOLD: Global Initiative for Chronic Obstructive Lung Disease. Odds ratios and $95 \%$ confidence intervals are displayed, as well as a pooled effect estimate weighted according to standard errors. 


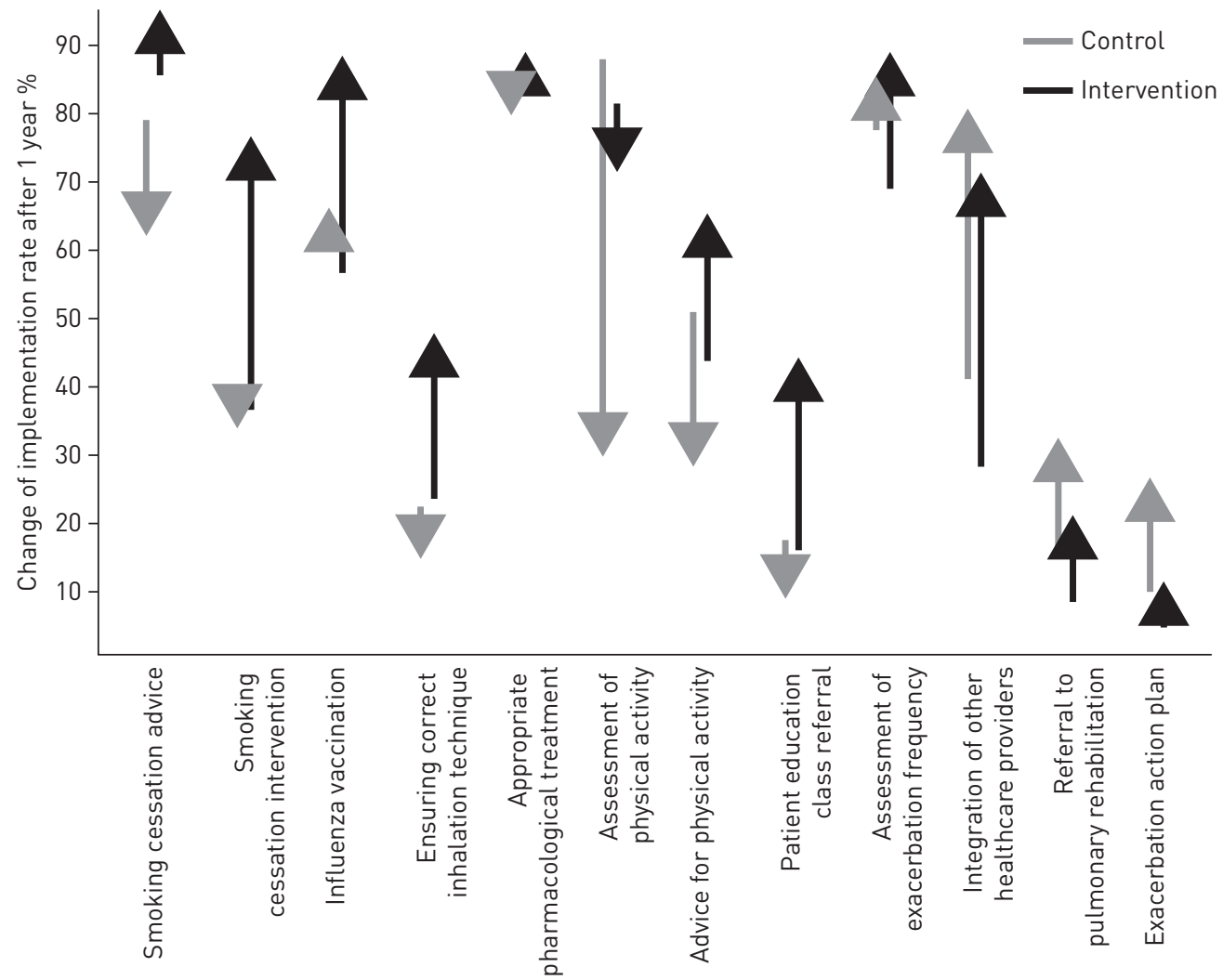

FIGURE 3 Net changes in implementation rates of individual key elements of chronic obstructive pulmonary disease care. Arrows point from the value at baseline to the value 1 year after the intervention.

care bundle was not used at all. To further explore the intervention's effects on health service utilisation, we assessed the 1-year frequency of planned and emergency COPD-driven practice visits as well as emergency department stays and hospitalisations at T1. A significant between-group difference appeared in the median (IQR) number of planned practice visits (intervention group $3(0-4)$ versus control group 1 $(0-3) ; p=0.04)$, but not within the other modes of health service utilisation.

\section{Discussion}

This cluster-randomised trial showed that a multifaceted intervention introducing a COPD care bundle to general practice teams increased the implementation rates of key elements of care compared with usual care based on patient self-report and previous 12-month recall. The between-group difference in implemented key elements of care was composed of an almost equal net increase in the intervention group and net decrease in the control group. The intervention therefore increased implementation rates of some key elements, but also prevented the otherwise occurring attrition of others. More than two-thirds of the patients were in early disease stages; significant intervention effects on disease-specific quality of life (CAT score) were not observed after 1 year.

Care bundles are effective on relevant outcomes such as disease progression, quality of life or exacerbation rates in hospital-based COPD care [28]. In our study, we detected significantly improved implementation of key elements recommended by guidelines and based on robust evidence [15-25,27]. We were unable to detect a direct impact on quality of life. However, owing to early disease stages and the slowly progressing natural course of the disease, a longer surveillance period may be required to demonstrate effects on patient outcomes. Yet, particularly in early disease stages of COPD, interventions retain the greatest potential for effects and should therefore be the cornerstone of care [14, 34]. In this context, it is noteworthy that we found the largest effects of our intervention for measures with strong evidence for improving prognosis, including smoking cessation interventions, physical activity promotion, patient education and influenza vaccination.

Complexity of care is associated with variation of care, and integrated standardised pathways of care are advocated to improve quality and outcomes [35]. Integration of care brings potential benefits to COPD patients and initiatives aim at fostering integrated care approaches in disease management for COPD [27, 
36]. Integrated care is, however, an umbrella term for heterogeneous components of care organisation and not an unambiguously defined one-size-fits-all model [37]. Interestingly, the recent and so far largest trial testing integrated care for COPD by KRUIs et al. [38] found no relevant effects on patient outcomes. This does not question integrated care in general, but it illustrates that little is known about the effect of the individual components aligning under the term. In this study, we promoted horizontal integration (i.e. redesigning COPD workflows handled by GPs and practice assistants), and implementing a care bundle as a pragmatic, flexible and collaborative disease management aid. The significant increase of planned consultations in the intervention group can be interpreted as redesign towards more proactive care culture in the targeted practices [39].

We regard this study as a first and promising COPD care bundle implementation trial in general practice. However, subsequent research in the field is needed to better understand the potential of this approach. A direct integration of the care bundle in electronic medical records may increase its adoption by physicians and further contribute to closing the gap in general practice health service delivery for COPD patients. Furthermore, relevant outcomes should be directly measured, but longer surveillance periods are required to enable this. The number of patients withdrawing from the study may be related to dissatisfaction with intensified healthcare delivery, possibly mediated by increasing costs and time expenditures. In Switzerland, only healthcare costs exceeding a patient-dependent minimum are reimbursed by statutory health insurance, therefore increased financial expenditures may have indeed contributed to dissatisfaction of a minority of patients. This effect may vary strongly according to financial coverage in different countries. The patient experience of the intervention should be examined and the costs associated with any benefits gained should be considered before conclusions about net benefits of intensified disease management can be drawn.

\section{Strengths and limitations of the study}

Some strengths and limitations must be mentioned. To the best of our knowledge, this is the first report of a COPD care bundle implementation in general practice with a cluster-randomised design. So far in this context the only available evidence was derived from hospital care or from general practice studies with different disease management interventions [28-30]. Another strength lies in the outcome assessment at the patient side: patient-recalled processes of care presumably reflect the successfully delivered elements of care better than the nonrecalled ones. However, this implies the limitation that the trial presumably underestimates the actually delivered elements of care. This potential recall bias does not invalidate the between-group difference we detected. The trial's open-label design is clearly a limitation to the study. In the control group, GPs might have felt discouraged knowing about their allocation to the usual care group, biasing the between-group difference in favour to the intervention group. In addition, there is a risk for contamination bias because half of the patients were treated in group practices where GPs from both study arms were colocated. Contamination, however, would have biased our results towards zero and therefore rather strengthens our positive findings. The significantly higher dropout rate in the intervention group is another important concern: even if reasons for dropout were similar it is still possible that a subgroup of patients felt uncomfortable with intensified healthcare provided in the intervention group, leading to undesirable self-deprivation from medical care. Lastly, despite randomisation, we found a small but statistically significant difference in disease severity variables between the study groups, with the intervention group being less severely affected by COPD. We believe, however, that the influence on disease management originating from this difference would have most likely resulted in intensified treatment in the more severely ill control group, again strengthening the trial's positive finding. Ultimately, we must keep in mind that we attribute the study effects to a multifaceted and therefore "impure" intervention. In addition to the care bundle or the team approach, other factors delivered to the intervention group during the workshops (mainly knowledge about the key elements of COPD care) may have been important active components in the trial.

\section{Conclusions}

A disease management intervention for general practice care teams introducing a COPD care bundle increased the adherence to recommended key elements of care. Subsequent beneficial effects on relevant patient outcomes are plausible but may require years until they become apparent given the insidious disease progression and the early disease stages of COPD patients in general practice.

Acknowledgements: Our thanks go to the practice teams that contributed to this study as well as to S. Groth (Study Nurse, Institute of Primary Care, University and University Hospital of Zurich, Zurich, Switzerland) who supported the study by conducting outreach telephone calls and providing technical assistance. We also thank I. Gravestock (Horten Center for Patient Oriented Research and Knowledge Transfer, Zurich, Switzerland) for editing the manuscript as a native English speaker. 
Author contributions: C. Steurer-Stey, T. Rosemann and K. Dalla-Lana conceived and designed the study. S. Markun, C. Steurer-Stey and K. Dalla-Lana acquired the data. S. Markun and C. Steurer-Stey analysed and interpreted the data, and drafted the manuscript to be revised critically by T. Rosemann and K. Dalla-Lana. S. Markun, T. Rosemann, K. Dalla-Lana and C. Steurer-Stey approved the final version to be published and agree to be accountable for all aspects of the study.

Conflict of interest: S. Markun, T. Rosemann, K. Dalla-Lana and C. Steurer-Stey report receiving grants from AstraZeneca Schweiz, Boehringer Ingelheim Switzerland, the Dept of Health of the Canton of Zurich, the Swiss Federal Office of Public Health (BAG) and Novartis Schweiz, during the conduct of the study.

Support statement: This project was supported by grants from the Swiss Federal Office of Public Health (BAG), the Swiss Medical Association (FMH) and the Dept of Health of the Canton of Zurich, and by unrestricted grants for Chronic Care and Patient Education from AstraZeneca Switzerland, Boehringer Ingelheim Switzerland and Novartis Switzerland. Funding information for this article has been deposited with the Crossref Funder Registry.

\section{References}

1 Wang H, Naghavi M, Allen C, et al. Global, regional, and national life expectancy, all-cause mortality, and cause-specific mortality for 249 causes of death, 1980-2015: a systematic analysis for the Global Burden of Disease Study 2015. Lancet 2016; 388: 1459-1544.

2 Buist AS, McBurnie MA, Vollmer WM, et al. International variation in the prevalence of COPD (the BOLD Study): a population-based prevalence study. Lancet 2007; 370: 741-750.

3 Global Initiative for Chronic Obstructive Lung Disease. Global Strategy for the Diagnosis, Management, and Prevention of Chronic Obstructive Pulmonary Disease. 2017. http://goldcopd.org/gold-2017-global-strategydiagnosis-management-prevention-copd/ Date last accessed: February 2, 2017.

4 Russi EW, Karrer W, Brutsche M, et al. Diagnosis and management of chronic obstructive pulmonary disease: the Swiss guidelines. Official guidelines of the Swiss Respiratory Society. Respiration 2013; 85: 160-174.

5 Jochmann A, Neubauer F, Miedinger D, et al. General practitioner's adherence to the COPD GOLD guidelines: baseline data of the Swiss COPD Cohort Study. Swiss Med Wkly 2010; 140: w13053.

6 Salinas G, Williamson C, Kalhan R, et al. Barriers to adherence to chronic obstructive pulmonary disease guidelines by primary care physicians. Int J Chron Obstruct Pulmon Dis 2011; 6: 171-179.

7 Johnston KN, Young M, Grimmer-Somers KA, et al. Why are some evidence-based care recommendations in chronic obstructive pulmonary disease better implemented than others? Perspectives of medical practitioners. Int $J$ Chron Obstruct Pulmon Dis 2011; 6: 659-667.

8 Steurer-Stey C, Dallalana K, Jungi M, et al. Management of chronic obstructive pulmonary disease in Swiss primary care: room for improvement. Qual Prim Care 2012; 20: 365-373.

9 Kaufmann C, Markun S, Hasler S, et al. Performance measures in the management of chronic obstructive pulmonary disease in primary care - a retrospective analysis. Praxis 2015; 104: 897-907.

10 Belletti D, Liu J, Zacker C, et al. Results of the CAPPS: COPD - assessment of practice in primary care study. Curr Med Res Opin 2013; 29: 957-966.

11 Brill SE, El-Emir E, Allinson JP, et al. Community-based recruitment of patients with COPD into clinical research Thorax 2014; 69: 951-952.

12 Markun S, Rosemann T, Dalla-Lana K, et al. The impact of case finding on the recruitment yield for COPD research in primary care: an observational study. Respiration 2016; 92: 308-315.

13 Kruis AL, Ställberg B, Jones RCM, et al. Primary care COPD patients compared with large pharmaceutically-sponsored COPD studies: an UNLOCK validation study. PLoS One 2014; 9: e90145.

14 Vasankari TM, Impivaara O, Heliövaara M, et al. No increase in the prevalence of COPD in two decades. Eur Respir J 2010; 36: 766-773.

15 van Eerd EA, van der Meer RM, van Schayck OC, et al. Smoking cessation for people with chronic obstructive pulmonary disease. Cochrane Database Syst Rev 2016; 8: CD010744.

16 Poole PJ, Chacko E, Wood-Baker RW, et al. Influenza vaccine for patients with chronic obstructive pulmonary disease. Cochrane Database Syst Rev 2006; 1: CD002733.

17 Kew KM, Mavergames C, Walters JA. Long-acting beta 2-agonists for chronic obstructive pulmonary disease. Cochrane Database Syst Rev 2013; 10: CD010177.

18 Appleton $\mathrm{S}$, Jones $\mathrm{T}$, Poole $\mathrm{P}$, et al. Ipratropium bromide versus long-acting beta-2 agonists for stable chronic obstructive pulmonary disease. Cochrane Database Syst Rev 2006; 3: CD006101.

19 Yang IA, Clarke MS, Sim EH, et al. Inhaled corticosteroids for stable chronic obstructive pulmonary disease. Cochrane Database Syst Rev 2012; 7: CD002991.

20 Barr RG, Bourbeau J, Camargo CA Jr. Tiotropium for stable chronic obstructive pulmonary disease. Cochrane Database Syst Rev 2005; 2: CD002876.

21 Press VG, Arora VM, Shah LM, et al. Teaching the use of respiratory inhalers to hospitalized patients with asthma or COPD: a randomized trial. J Gen Intern Med 2012; 27: 1317-1325.

22 McCarthy B, Casey D, Devane D, et al. Pulmonary rehabilitation for chronic obstructive pulmonary disease. Cochrane Database Syst Rev 2015; 2: CD003793.

23 Paneroni M, Simonelli C, Vitacca M, et al. Aerobic exercise training in very severe chronic obstructive pulmonary disease: a systematic review and meta-analysis. Am J Phys Med Rehabil 2017; 96: 541-548.

24 Cannon D, Buys N, Sriram KB, et al. The effects of chronic obstructive pulmonary disease self-management interventions on improvement of quality of life in COPD patients: a meta-analysis. Respir Med 2016; 121: 81-90.

25 Zwerink M, Brusse-Keizer M, van der Valk PD, et al. Self management for patients with chronic obstructive pulmonary disease. Cochrane Database Syst Rev 2014; 3: CD002990.

26 Mitchell KE, Johnson-Warrington V, Apps LD, et al. A self-management programme for COPD: a randomised controlled trial. Eur Respir J 2014; 44: 1538-1547.

27 Kruis AL, Smidt N, Assendelft WJ, et al. Integrated disease management interventions for patients with chronic obstructive pulmonary disease. Cochrane Database Syst Rev 2013; 10: CD009437. 
Ospina MB, Mrklas K, Deuchar L, et al. A systematic review of the effectiveness of discharge care bundles for patients with COPD. Thorax 2017; 72: 31-39.

29 Zwar NA, Hermiz O, Comino E, et al. Care of patients with a diagnosis of chronic obstructive pulmonary disease: a cluster randomised controlled trial. Med J Aust 2012; 197: 394-398.

30 Rea H, McAuley S, Stewart A, et al. A chronic disease management programme can reduce days in hospital for patients with chronic obstructive pulmonary disease. Intern Med J 2004; 34: 608-614.

31 Steurer-Stey C, Markun S, Lana KD, et al. The improving care in chronic obstructive lung disease study: CAROL improving processes of care and quality of life of COPD patients in primary care: study protocol for a randomized controlled trial. Trials 2014; 15: 96.

32 Grimshaw JM, Shirran L, Thomas R, et al. Changing provider behavior: an overview of systematic reviews of interventions. Med Care 2001; 39: II2-II45.

33 Grol R, Wensing M. What drives change? Barriers to and incentives for achieving evidence-based practice. Med $J$ Aust 2004; 180: S57-S60.

34 Josephs L, Culliford D, Johnson M, et al. Improved outcomes in ex-smokers with COPD: a UK primary care observational cohort study. Eur Respir J 2017; 49: 160211.

35 Institute of Medicine Committee on Quality of Health Care in America. Crossing the Quality Chasm: A New Health System for the 21st Century. Washington, National Academies Press, 2001.

36 Bousquet J, Addis A, Adcock I, et al. Integrated care pathways for airway diseases (AIRWAYS-ICPs). Eur Respir J 2014; 44: 304-323.

37 Suter E, Oelke ND, Adair CE, et al. Ten key principles for successful health systems integration. Healthc Q 2009; 13: 16 .

38 Kruis AL, Boland MRS, Assendelft WJJ, et al. Effectiveness of integrated disease management for primary care chronic obstructive pulmonary disease patients: results of cluster randomised trial. BMJ 2014; 349 : g5392.

39 Fromer L. Implementing chronic care for COPD: planned visits, care coordination, and patient empowerment for improved outcomes. Int J Chron Obstruct Pulmon Dis 2011; 6: 605-614. 